\title{
Direct Observation and Control of Supported \\ Lipid Bilayer Formation with Interferometric
}

\section{Scattering Microscopy}

Joanna Andrecka, Katelyn M. Spillane, Jaime Ortega-Arroyo, and Philipp Kukura*

Physical and Theoretical Chemistry Laboratory,

Department of Chemistry, University of Oxford, South Parks Road,

Oxford, OX1 3QZ, UK

\section{KEYWORDS}

Supported lipid bilayer formation, interferometric scattering microscopy, label-free optical imaging 


\begin{abstract}
Supported lipid bilayers (SLB) are frequently used to study processes associated with or mediated by lipid membranes. The mechanism by which SLBs form is a matter of debate, largely due to the experimental difficulty associated with observing the adsorption and rupture of individual vesicles. Here, we used interferometric scattering microscopy (iSCAT) to directly visualize membrane formation from nanoscopic vesicles in real time. We observed a number of previously proposed phenomena such as vesicle adsorption, rupture, movement and a wave-like bilayer spreading. By varying the vesicle size and the lipid-surface interaction strength, we rationalized and tuned the relative contributions of these phenomena to bilayer formation. Our results support a model where the interplay between bilayer edge tension and the overall interaction energy with the surface determine the mechanism of SLB formation. The unique combination of sensitivity, speed and label-free imaging capability of iSCAT provides exciting prospects not only for investigations of SLB formation, but also for studying assembly and disassembly processes on the nanoscale with previously unattainable accuracy and sensitivity.
\end{abstract}
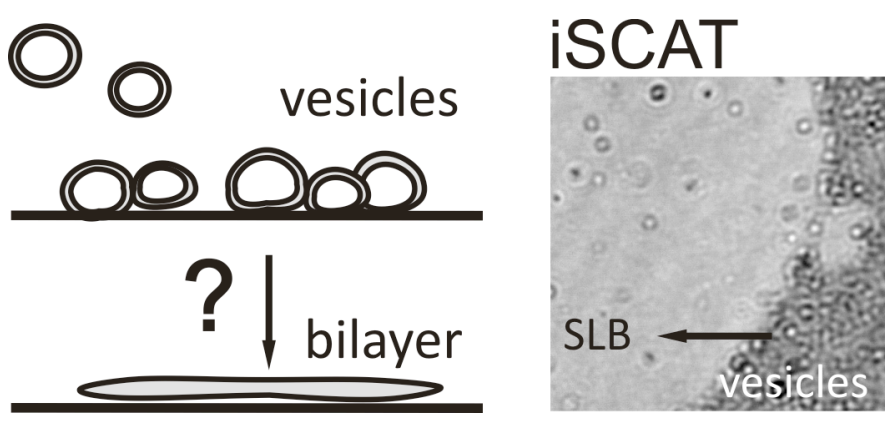
Supported lipid bilayers (SLB) formed by vesicle adsorption and rupture serve as an ideal system to study the structural transition between nanoscopic vesicles and a mesoscopic bilayer membrane. This reorganization of lipid assemblies is fundamental to our understanding of a series of biological processes, such as endo- and exocytosis, cell signalling and cellular transport. ${ }^{1-4}$ As a consequence, the mechanism of membrane formation has been studied extensively over the past decades. ${ }^{5}$ It is commonly accepted that vesicle adsorption onto a solid substrate precedes SLB formation, but many aspects of the nature and sequence of the subsequent events remain unclear. ${ }^{6-9}$ For instance, while atomic force microscopy (AFM) has provided direct evidence that isolated vesicles can rupture to form bilayer disks ${ }^{10}$ in support of theoretical predictions, ${ }^{11-13}$ quartz crystal microbalance resonant frequency and dissipation (QCM-D) combined with surface plasmon resonance (SPR) spectroscopy has suggested that vesicle rupture occurs only after a large number of intact vesicles have adsorbed on the surface, a phenomenon defined as the critical coverage. ${ }^{14}$

To further investigate the behavior of nanoscopic vesicles upon adsorption more complex experimental approaches were used, often combining two or more techniques including AFM, QCM-D, SPR, ellipsometry and fluorescence microscopy. ${ }^{15-19}$ In addition, the effect of various experimental conditions on SLB formation was investigated, such as lipid charge ${ }^{8,15,16}$ surface charge, ${ }^{20-22}$ vesicle size,${ }^{10,21,23}$ temperature, ${ }^{24}$ ionic strength, ${ }^{7,15,16,21} \mathrm{pH}^{7,25}$ and osmotic pressure. ${ }^{26}$ Taken together, these studies suggested the presence of additional phenomena such as the existence of active bilayer edges that catalyze membrane formation, ${ }^{27,28}$ mobility of adsorbed vesicles, ${ }^{16,28}$ vesicle fusion or partial rupture ${ }^{17}$ and desorption of lipid vesicles in the final stages of SLB formation (Figure 1). ${ }^{28,29}$ In all cases, however, these processes had to be inferred as the direct observation of individual vesicles was not possible. 
The phenomena outlined above cannot easily be reconciled into a single model for SLB formation. The current discrepancies are largely due to fundamental experimental limitations. For instance AFM can image individual vesicles, but is slow on the timescale of membrane formation and invasive due to the interaction of the cantilever tip with the sample. ${ }^{30}$ Combination of ensemble-based techniques such as QCM-D or SPR with AFM, intended to bridge the gap between nanoscopic and ensemble technique questioned the relationship between surface coverage and the QCM-D signal, thereby challenging the interpretation and meaning of the bulk results. ${ }^{31}$ Moreover, the broad range of experimental conditions make it difficult to compare data obtained using different techniques. Recent studies using fluorescence microscopy have added to the confusion by proposing a different bilayer formation mechanism altogether, where vesicle affinity to bilayer edges plays an important role. ${ }^{28}$ In addition, the use of fluorophores, required to visualize vesicles smaller than $200 \mathrm{~nm}$, comes at the expense of altering the lipid properties and vesicle stability. ${ }^{32-34}$

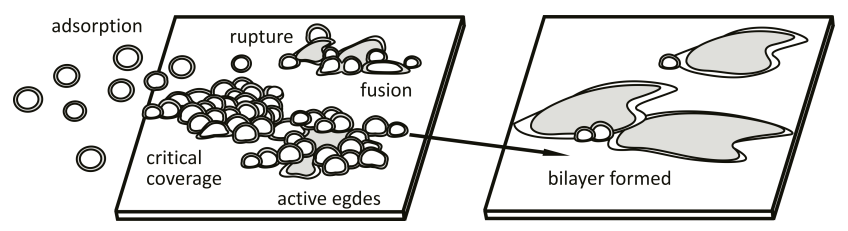

Figure 1. Schematic of phenomena proposed to contribute to the mechanism of SLB formation. Bilayer formation begins with the adsorption of nanoscopic vesicles to the surface followed by spontaneous rupture or vesicle fusion due to surface-vesicle and direct vesiclevesicle interaction. If vesicles do not rupture spontaneously they may reach a critical coverage after which rupture of a small fraction of vesicle leads to the formation of active 
edges. The SLB forms via a mechanism governed either by spontaneous rupture or triggered by a critical coverage of vesicles.

Elucidating the mechanism of SLB formation thus calls for an approach that is non-invasive, capable of visualizing individual vesicles, sufficiently sensitive to observe adsorption and rupture events and fast enough to capture the membrane formation process in real time. Here, we demonstrate that interferometric scattering microscopy (iSCAT) ${ }^{35-39}$ satisfies all of the above criteria and use it to elucidate the nanoscopic mechanism of SLB formation. We show that bilayer formation is accompanied by vesicle motion, diffusion and a wave-like propagation. By varying the interaction strength between the substrate and the adsorbed vesicles we demonstrate that all of the above processes contribute to the process, but in a manner highly dependent on the interplay between edge tension and the lipid-surface adhesion energy.

\section{RESULTS}

\section{iSCAT as a tool to visualize SLB formation}

iSCAT relies on efficiently detecting light scattered by nanoscopic labels. In principle, it involves no more than collecting the light scattered by a particle together with a reference light field, often provided by the weak reflection at an interface (Figure 2A). In the presence of a scatterer, in this case a lipid vesicle, the total intensity of light returning into the direction of the incident beam will be different than in the absence of that object. The use of a coherent light source improves the interference contrast, but also suffers from additional backreflections that cause speckle and background. To optimize the illumination, we pass the illumination beam through acousto optic deflectors (AODs) and image the resulting beam 
displacement with telescopic lenses into the back-focal plane of the imaging objective after passing through a polarizing beam splitter (Figure 2B). Addition of a quarter wave plate ensures that all scattered and reflected light collected by the microscope objective is then reflected by the beam splitter before being imaged onto a fast CMOS camera. For rapid beam scanning $(>40 \mathrm{kHz})$, the illuminated area appears uniform on the timescale of the camera exposure (40 ms). A second laser beam is used to stabilize the objective-sample distance by monitoring the reflection at the cover slip surface using a bi-plane approach. ${ }^{40}$
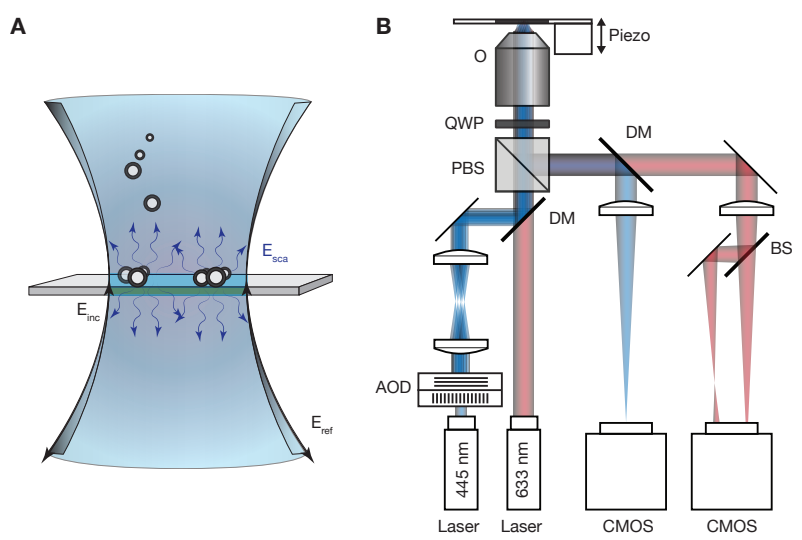

Figure 2. Interferometric scattering microscopy (iSCAT). (A) Schematic of incident, reflected and scattered light fields upon adsorption of individual vesicles on a substrate. (B) Experimental setup. O: microscope objective, QWP: quarter-wave plate, PBS: polarizing beam splitter, DM: dichroic mirror, BS: 50-50 beam splitter, AOD: acousto optic deflector. The diode laser at $445 \mathrm{~nm}$ is used for iSCAT imaging, while a Helium-Neon laser at $633 \mathrm{~nm}$ is used for focus-stabilization implemented by a bi-plane imaging approach on a second CMOS camera. ${ }^{40}$ In brief, the spot-sizes are fit to Gaussians and the extracted widths used as as feedback for the piezo-stage to stabilize the objective-sample distance to within $20 \mathrm{~nm}$. The two imaging colors are combined and separated by dichroic mirrors.

\section{SLB formation from large unilamellar vesicles}


The experiment begins with a cleaned microscope cover glass acting as the solid substrate for the SLB. In an iSCAT microscope, this substrate produces a weak background signal reminiscent of a speckle pattern caused by its nanoscopic roughness (Figure $3 \mathrm{~A}){ }^{41}$ To initiate membrane formation, we added a solution containing unilamellar DOPC vesicles extruded through polycarbonate membranes with nominally $100 \mathrm{~nm}$ pores (extruded unilamellar vesicles, EUVs). At our imaging speed of $25 \mathrm{~Hz}$, bright, diffraction limited spots appeared immediately upon the addition of the vesicle solution (Figures 3B-C). We emphasize that the contrast of the vesicles can be tuned from positive to negative by moving the sample over a few hundred $\mathrm{nm}$ through the focus by taking advantage of the rapidly changing Gouy phase. ${ }^{42}$ We chose a positive contrast here, but all experiments could have equally been performed with a negative iSCAT contrast. Close inspection of Figure $3 \mathrm{C}$ reveals adsorbed vesicles in addition to nanoscopic bilayer patches in the form of on-spherical microscopic features that are brighter than the image background. As the vesicle coverage increased, more of these structures appeared, transformed into larger, bright patches (Figure 3D, E) and eventually combined to generate a continuous bilayer. During this process some vesicles became mobile and ruptured at a different position, or desorbed and diffused on top of the membrane (Figure 3F). We did not observe any binding of vesicles after bilayer formation likely due to the lower binding affinity of the vesicles to the bilayer compared to the bare glass surface.

To better understand the transformation from adsorbed vesicles to bilayer patches and eventually the continuous bilayer (Supporting Information, Movie S1), we focused on the highlighted area in Figure 3C (Supporting Information, Movie S1-A), with snapshots of key steps shown in Figure 3G. During the first frames (Figure 3G), the adsorbed vesicle marked with a black arrow disappeared, and was replaced by a non-spherical structure of higher iSCAT contrast than the background (Figure 3G, 2). Following the adsorption of another vesicle (Figure 3G, 3) and its subsequent rupture, this structure grew into a larger, smooth 
patch as indicated by the black line (Figure 3G, 4). A plot of iSCAT contrast as a function of time at the position marked with a white arrow in Figure $3 \mathrm{G}$, shows the adsorption of a single vesicle, followed by its rupture to form a bilayer (Figure $3 \mathrm{H}$ ). The latter produced a different iSCAT contrast compared to the wet substrate due to the differing reflectivity of a glassbilayer-water $(\mathrm{n}=1.52,1.48,1.33)$ and a bare-glass water interface $(\mathrm{n}=1.52,1.33)$. The corresponding time-trace for a departing, rather than a rupturing vesicle exhibited distinctly different behavior (Figure 3J).

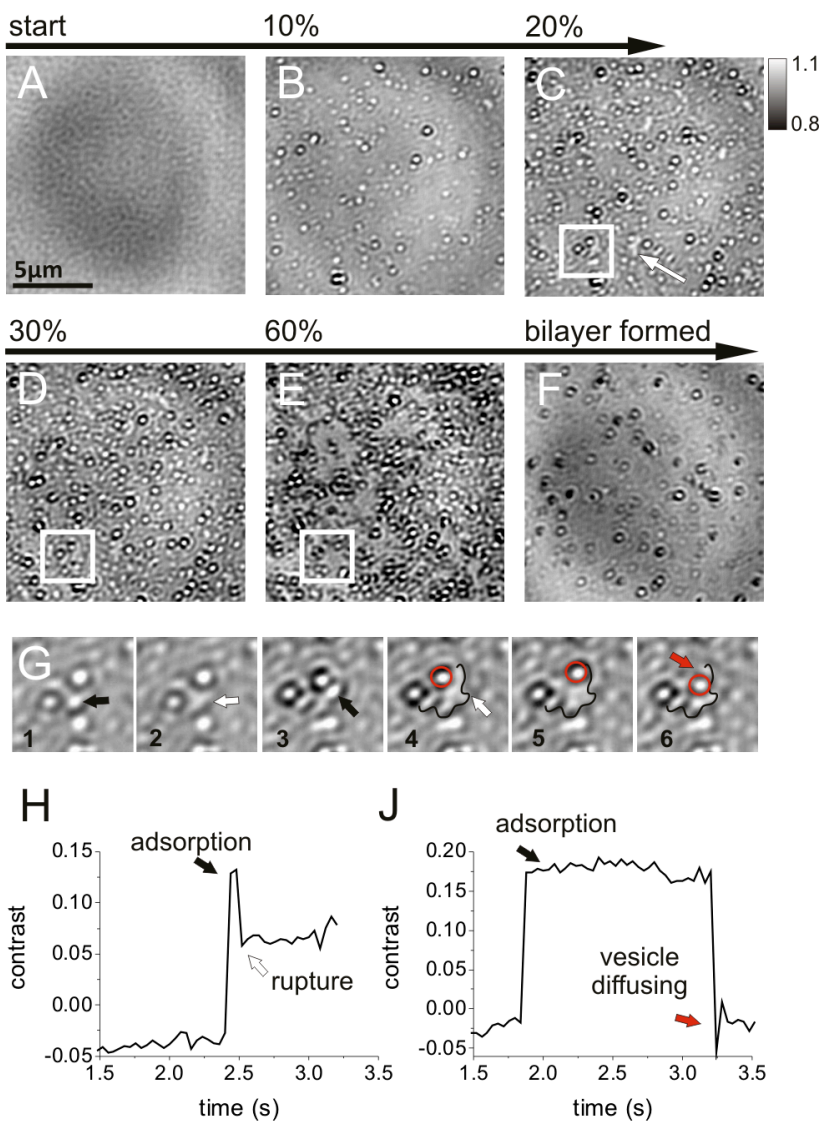

Figure 3. Bilayer formation from extruded $100 \mathrm{~nm}$ vesicles on plasma-cleaned glass as a solid substrate. (A) iSCAT image of a clean glass cover slip in buffer. (B) Adsorption of individual vesicles after addition of a vesicle solution to the same area shown in (A) with membrane formation $10 \%$ completed $(\sim 1.75 \mathrm{~s})$. We define the completion time as the interval 
from the adsorption of the first vesicle to full bilayer formation within the field of view $(\sim 17.5 \mathrm{~s})$. (C) The same area but at $20 \%$ completion $(\sim 3.5 \mathrm{~s})$. Arrows indicate the appearance of enlarged nanoscopic patches of bright and uniform contrast. (D-F) Final stages of bilayer formation at 30,60 and 100\% completion. (G) Evolution of the nanoscopic patches observed in the boxed region in $\mathrm{C}$ with adsorption of vesicles (black arrow, 1 and 3) and growth of a smooth structure from single adsorbed vesicles (white arrow, 2 and 4). The $z$ scale represents the signal contrast with the baseline corresponding to 1 . (H) Time-trace for the iSCAT contrast of a single pixel in the center of the particle marked with the black arrow shown in the first image of $(\mathrm{G})$. $(\mathrm{J})$ Corresponding time-trace for the particle marked with the red circle which starts diffusing after the final image shown in $(\mathrm{G})$. The entire process is shown in Movies S1 and S1-A (Supporting Information).

\section{Wave-like spreading of SLBs}

We repeated the measurements shown in Figure 3 using smaller diameter $(20 \mathrm{~nm})$ sonicated unilamellar vesicles (SUV) to investigate the effect of vesicle size on the mechanism of bilayer formation. As for larger EUVs, sonicated vesicles adsorbed to the surface (Figure 4A), but the formation of bilayer patches began at a much later stage (Figure 4B) and was quickly followed by completion of the SLB (Figure 4C). During this last stage of membrane formation, we observed a wave-like propagation of the bilayer (Figure 4D). Defining the duration of SLB formation as the time elapsed from the adsorption of the first vesicle to the completion of the bilayer, the wave-like propagation of the bilayer was limited to the last $10 \%$ of the process (Supporting Information, Movie S2). 
The snapshots in Figure 4D demonstrate that the bilayer propagates along existing edges but does not provide any details about the nanoscopic events leading to bilayer formation. By subtracting consecutive frames, we can reveal the arrival and departure of individual vesicles, irrespective of the number of vesicles present on the surface (Figure 5A). In the differential image bright, spherically symmetric signals correspond to vesicle adsorption, while dark features are due to vesicle rupture. Vesicle motion from frame to frame caused asymmetric signatures containing both bright and dark features (Figure 5A, white star) and out-of-focus signals (grey arrow) from diffusing vesicles that had not yet bound to the. The signal-tonoise-ratio (SNR) of the differential images surpassed that of the raw images, because the background was constant and the only residual fluctuations are caused by shot noise in the detection limited by the magnification and the well depth of the CMOS camera $(0.3 \%$ RMS). ${ }^{36}$ At the frame rate chosen in these experiments, unbound vesicles diffused sufficiently fast to produce a negligible and blurred signal in the differential images. Furthermore, the strong dependence of the iSCAT signal on the distance between the vesicle and the surface ${ }^{43}$ reduced any signatures due to diffusing vesicles beyond the sensitivity limit of the measurement. We thus conclude that iSCAT in our current experimental configuration only detected surface bound vesicles.

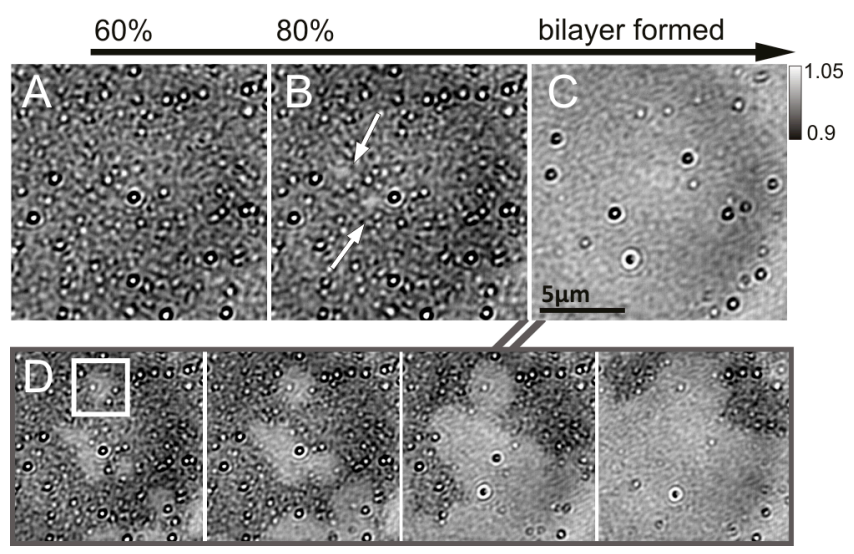


Figure 4. Bilayer formation from sonicated unilamellar vesicles $(\sim 20 \mathrm{~nm})$ on plasma-cleaned glass. (A-C) Surface at 60, 80 and $100 \%$ completion. White arrows in (B) indicate the appearance of bilayer patches which rapidly grow, coalesce and eventually form the entire bilayer with few vesicles diffusing on the membrane. (D) Representative snapshots highlighting the membrane formation process between (B) and (C). Every eighth frame (200 ms) is shown for clarity.

Applying the image subtraction procedure to Figure 4D allowed us to investigate the nanoscopic dynamics of bilayer propagation on a vesicle-by-vesicle basis. The differential images shown in Figure 5B-G originate from the development and evolution of the small bilayer patch marked with a box in Figure 4D. The process began with the rupture of a seed vesicle in the center of the image (Figure 5B), followed by the simultaneous rupture of the nearest neighbouring vesicles leading to a circular patch (Figure 5C). The circular patch spread, with another propagating bilayer edge entering the image from the bottom left (Figure 5D, E). The two edges met (Figure 5F) and disappeared as they coalesced into one large bilayer patch (Figure 5G).

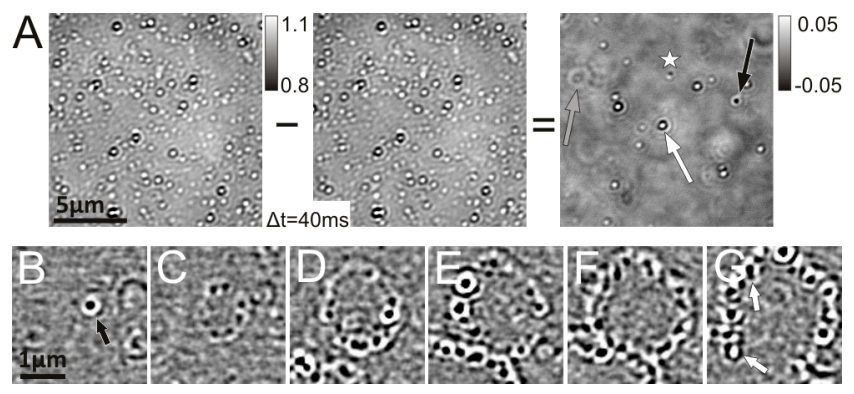


Figure 5. Bilayer formation analysis by consecutive image subtraction. (A) Subtraction of subsequent frames reveals: adsorbed vesicles (bright circularly symmetric PSFs, white arrow), ruptured/departed vesicles (dark circularly symmetric PSFs, black arrow), diffusing vesicles in solution (large blurred and defocused PSFs, throughout the whole image, one of them marked with a grey arrow) and vesicle movement in the image plane (narrow asymmetric PSFs, white star). (B-G) Differential image analysis of the boxed region in figure 4D. After initial rupture of a single seed vesicle (B), more vesicles rupture at the edge of the growing bilayer $(\mathrm{C}-\mathrm{F})$. The differential images from B-C reveal circularly symmetric PSFs at the edge of the propagating bilayer. Then, asymmetric PSFs develop at the edge of the propagating bilayer indicated by white arrows $(F, G)$.

\section{Analysis of spontaneous vs. wave-induced vesicle rupture}

Our results not only provide a visual picture of the nanoscopic mechanism of membrane formation, but also enable quantitative analysis. The image subtraction procedure presented in Figure 5A generates images that are familiar from fluorescence-based super-resolution microscopy (S1-4, subtracted), where individual fluorophores are activated and then localized. ${ }^{44}$ In our case, the optical signal is not activated, but generated by the arrival and rupture of the vesicles. By applying single particle localization to differential images we isolated individual vesicles and produced histograms of iSCAT contrast. We found that EUVs with diameters of $100 \mathrm{~nm}$ produce predominantly 5\% contrast (Figure 6A), while the histogram for SUVs peaked at $<1 \%$ signal (Figure 6B).

Focusing on specific, nanoscopic areas of $(100 \mathrm{~nm})^{2}$, we generated time traces of the arrival and rupture of individual vesicles. We observed no discernible signal until vesicle adsorption produced a bright signal in the differential image and thus a positive spike in the time trace. 
As long as the vesicle remained in its original position and did not change its scattering properties, the differential signal fluctuated around zero. Upon rupture, the vesicle generated a negative spike of equal but opposite magnitude compared to vesicle adsorption (Figure 6C). We observed similar behavior for SUVs but with a reduced iSCAT contrast (Figure 6D).

Figure 6E summarizes the variation in the time delays between vesicle adsorption (filled circles) and rupture (empty circles). For all vesicles, the adsorption rate remained constant until the surface became crowded and tailed off as expected for a standard adsorption isotherm. For $100 \mathrm{~nm}$ EUVs, vesicles ruptured at all times but the rate increased after bilayer formation was $40 \%$ complete. For SUVs, however, almost all vesicles ruptured during the last $10 \%$ of the membrane formation process. Extruded vesicles of intermediate sizes $(30 \mathrm{~nm}$ and $50 \mathrm{~nm}$ ) exhibited a mixture of these behaviors (Supporting Information, Movies S3 and S4). During the early stages of SLB formation, vesicles ruptured more often for the intermediate EUVs than for SUVs, but eventually the rupture rate accelerated in both cases in a way that is not observed for the largest (100 nm) EUVs.

We remark that the time traces such as those shown in Figure 6 were only observable for a fraction of the detected vesicles as vesicle motion complicates the nature of the observed signals (Supporting Information, Movie S1 and S1-A). Although it was difficult to observe vesicle motion for SUVs due to their low iSCAT contrast and high density, their mobility could be deduced from the differential images. For instance, at the early stages of bilayer formation, vesicle rupture generated spherically symmetric PSFs (Figure 5B-5E). As the bilayer edge accelerated, however, the differential images exhibited asymmetric PSFs that appeared dark on the inside of the spreading bilayer and bright on the outer border (white arrows). Such a dispersive PSF is expected for a vesicle moving together with the spreading bilayer. The corresponding time trace for such vesicles exhibited vesicle adsorption but the information about vesicle rupture was lost. Such traces were not used for further analysis but 
the information available from the selected time traces shown in Figure $6 \mathrm{C}$ reproduces the qualitative picture (Supporting Information, Movie S1 - S4).
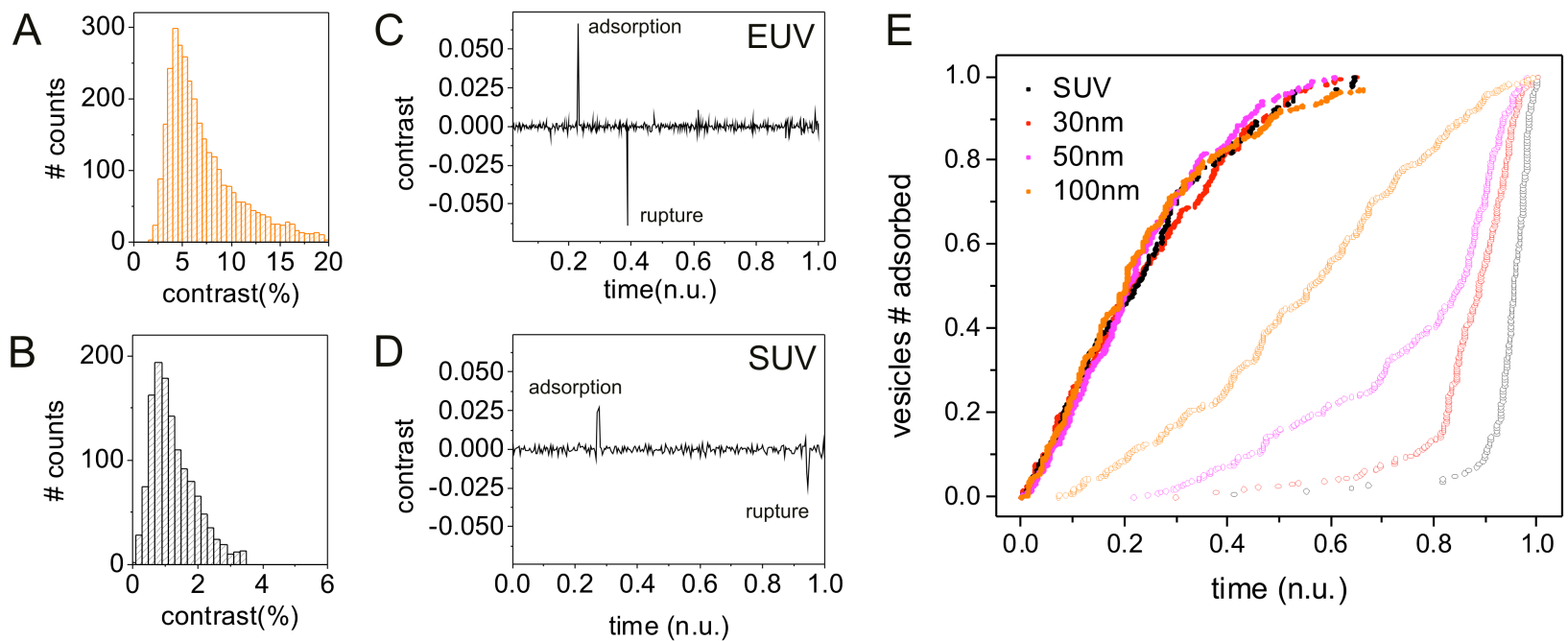

Figure 6. Analysis of membrane formation. (A, B) Histogram of iSCAT contrasts for adsorbed $100 \mathrm{~nm}$ EUVs and SUVs, respectively. (C) Detection of adsorption and rupture events in the same nanoscopic $(100 \mathrm{~nm})^{2}$ location as a function of time for $100 \mathrm{~nm}$ EUVs. (D) Equivalent trace for SUVs. (E) Number of adsorbed (filled circles) and ruptured vesicles (open circles) as function of bilayer completion. The number of adsorbed vesicles is given per unit area and normalized (in this case the field of view, $15 \times 15 \mu \mathrm{m}$ ), where 1 corresponds to the maximum number of vesicles adsorbed prior to membrane formation. To avoid bias from undetected vesicles, no absolute numbers were provided. Total number of time traces used: 142, 271, 352, 827 for SUVs, $30 \mathrm{~nm}, 50 \mathrm{~nm}$ and $100 \mathrm{~nm}$ EUVs, respectively.

\section{Influence of vesicles' size and shape in iSCAT signal}

To verify the observation of individual vesicles, it is useful to compare the observed iSCAT contrast for the vesicles used here with previous iSCAT studies of nanoscopic objects. 
Assuming a lipid area of $0.7 \mathrm{~nm}^{2}$ and a molecular weight of 790 Da per lipid yields a weight of $2.8 \mathrm{MDa}$ of material with a refractive index of $\sim 1.48$ per $20 \mathrm{~nm}$ vesicle. Given that a single SV40 VLP weighs $15.8 \mathrm{MDa}$, has a similar refractive index and produces $\sim 4 \%$ contrast in our current experimental configuration, we predict an iSCAT contrast for individual $20 \mathrm{~nm}$ vesicles of $0.7 \%$. Our histograms are slightly biased towards larger vesicles at the current level of baseline noise $(0.3 \%)$ due to a high contrast cut-off to avoid false positives. According to these considerations, $100 \mathrm{~nm}$ vesicles should produce an average signal of $17.5 \%$, rather than the observed $5 \%$. Although the origin of this discrepancy is currently unclear, there are two possible explanations. If the vesicle remains spherical upon adsorption, light scattered by the lipids on the vesicle side far away from the surface is no longer subject to the refractive index gradient at the interface known to direct light towards the high numerical aperture objective. ${ }^{45}$ Large vesicles thus scatter comparatively less light into the collection optic than very small vesicles $(\sim 20 \mathrm{~nm})$ making their contrast smaller than expected from pure polarizability considerations. Alternatively, if the vesicles flatten significantly upon adsorption to the surface, the effective refractive index of the environment becomes an average of glass and water (1.43) and thus very close to that of DOPC making the vesicle a poorer scatterer. In contrast, a small vesicle experiences a much larger barrier to distortion and thus remains largely surrounded by water with a refractive index of 1.33 . Finally, binding events that occur during the finite camera exposure time produce smaller than expected contrasts that can further reduce the measured iSCAT contrast. We remark, however, that the absolute magnitude of the observed iSCAT contrast has little consequences on the observed bilayer formation dynamics. The scope of this work was not to replace dynamic light scattering as a particle size characterization tool, but rather to reveal the mechanism of SLB formation. For sonicated vesicles we did not detect every particle that 
adsorbs to the surface, but could still observe the mechanism of bilayer formation with unprecedented clarity.

\section{Vesicle-surface interaction strength influences the mechanism of bilayer formation}

The results demonstrate that the relative contributions of wave-like bilayer spreading and individual vesicle rupture depend on the size of the adsorbed vesicles, but they do not reveal the underlying physical origin. We thus repeated our measurements on mica, a substrate where we observed formation of high-quality bilayers with different surface functionalities. Plasma treatment covers the mica surface with hydroxyl groups and changes the adhesion potential compared to the freshly cleaved surface. ${ }^{46}$ Samples prepared with SUVs on freshly cleaved mica resulted in even larger propagating bilayer waves compared to those on glass (Figure 7A, Supporting Information, Movie S5). For plasma-etched mica we observed a reduction in the size of the propagating bilayer wave with respect to freshly cleaved mica, resulting in movies similar to those obtained on plasma-cleaned glass shown in Figure 4D (Figure 7B, Supporting Information, Movie S6). Increasing the vesicle size to $100 \mathrm{~nm}$ drastically changed the dynamics of bilayer formation between glass and mica. In contrast to EUVs on glass, where individual vesicle rupture occurred frequently throughout the membrane formation process (Figure 3), EUVs on freshly cleaved mica formed bilayers in a wave-like fashion characteristic of SUVs on glass. We observed the formation of several microscopic patches (Figure 7C), but a large wave dominated the bilayer formation process. In addition, the larger vesicle size resulted in a higher iSCAT signal (Figure 6) and facilitated the observation of vesicle motion at the edge of the forming bilayer (Supporting Information, Movies S7A-B), which was difficult to observe for smaller vesicles. Compared to SUVs, however, EUVs formed bilayers about an order of magnitude more slowly. Upon plasma treatment of the mica surface, the membrane formation process reverted to the behavior 
originally observed on glass: multiple individual rupture events and nanoscopic bilayer patch formation (Figure 7D, Supporting Information, Movie S8).

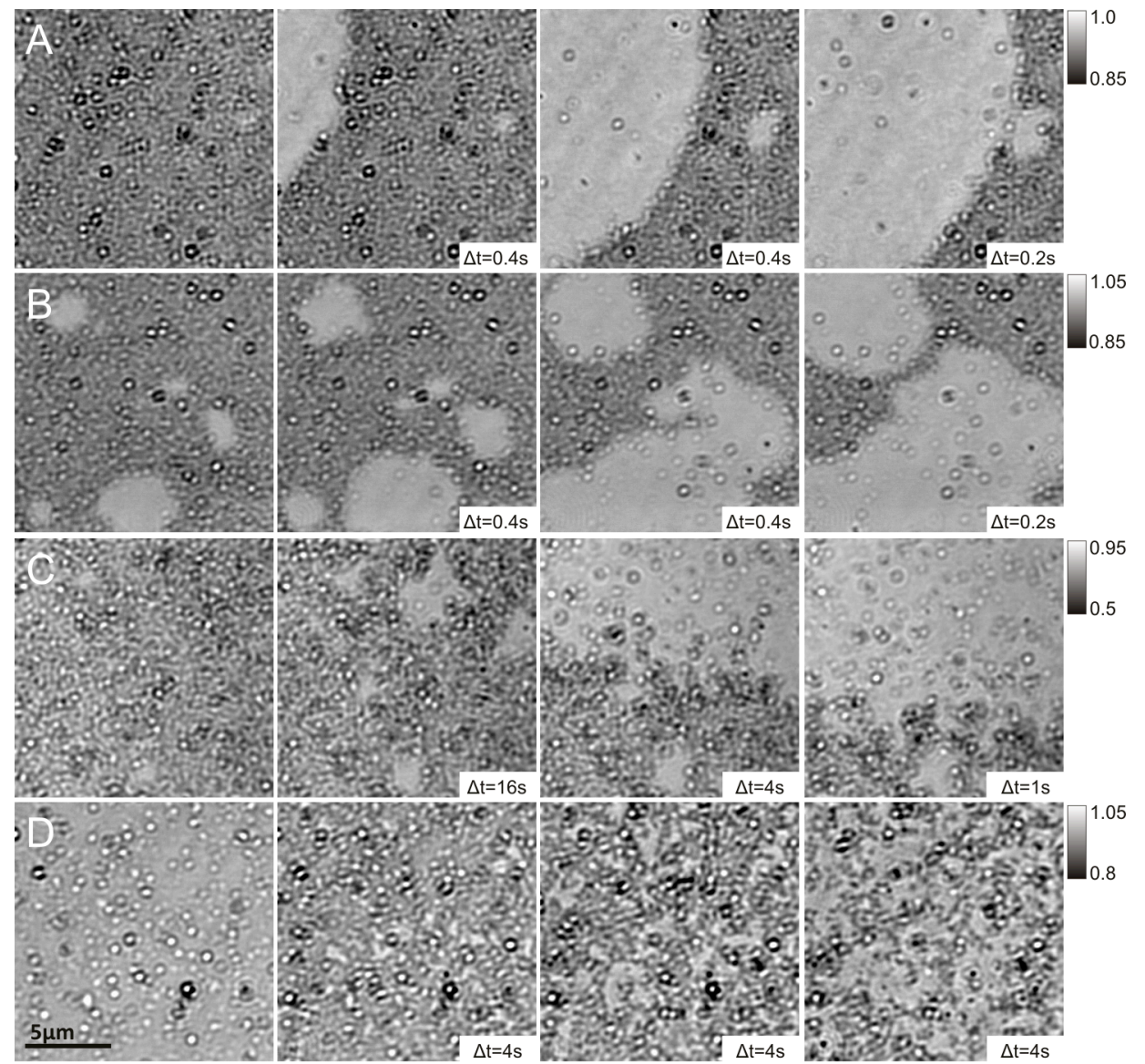

Figure 7. Bilayer formation on untreated and plasma-cleaned mica. The evolution of the supported lipid bilayer from SUVs on untreated (A) and on plasma-cleanedmica (B) exhibit analogous behavior to SUVs on glass (Figure 4). For $100 \mathrm{~nm}$ EUVs on untreated mica (C) vesicles rupture after surface coverage is complete. The same $100 \mathrm{~nm}$ EUV solution on plasma etched mica (D) causes the membrane formation process to follow a similar spontaneous rupture scenario as on glass (Figure 3).

\section{DISCUSSION AND CONCLUSIONS}


A variety of processes have been proposed to contribute to the mechanism of SLB formation although many of them have never been observed directly. In addition, their interplay, relative contribution, dependence on experimental conditions and role in the overall process, could not be combined into a single, consistent model. Here, we use iSCAT to directly visualize individual vesicle adsorption and the subsequent transformation into a planar bilayer for the first time. Importantly, we observe the entire formation process in a single recording and detect phenomena including wave-like spreading, spontaneous and driven vesicle rupture and vesicle diffusion. Thereby, we have identified and characterized the key steps of the SLB formation process. The rate of spontaneous vesicle rupture decreases with vesicle size, contrary to previous results suggesting that the qualitative behavior of adsorbed vesicles is size-independent. ${ }^{21}$ EUVs rupture spontaneously even at low surface coverage for strong interaction potentials with the substrate. For SUVs, however, vesicles rupture almost exclusively at the edge of the spreading bilayer wave, after the surface is covered by adsorbed vesicles. By reducing the interaction strength between the substrate and the vesicles, we reproduce this behavior with $100 \mathrm{~nm}$ EUVs. The transition from predominantly waveinduced rupture towards spontaneous vesicle rupture shown in Figure 7 suggests that the total interaction strength between adsorbed vesicles and the surface is a key factor in determining the mechanism of SLB formation. Our results also clarify the role of critical coverage in SLB formation. Close proximity of vesicles does not catalyze spontaneous vesicle rupture; otherwise the iSCAT movies of SLB formation would appear very similar for SUVs and EUVs on glass with the only difference being a later onset of nanoscopic patch formation for SUVs. The observed wave-like spreading suggests that the major role of critical coverage is to ensure efficient propagation of the bilayer. During the spread of the bilayer wave, we observed both vesicle rupture and, for the first time, physical motion of vesicles as they are being pushed along the bilayer edge. 
Although a detailed theoretical analysis is beyond the scope of this work, a qualitative discussion of our results in the light of the existing literature is insightful. The vesicle-surface interaction energy scales with the adhesion potential and the contact area between the vesicle and the surface and leads to vesicle rupture if it is sufficiently large. ${ }^{12}$ As a result, vesicles turn into nanoscopic bilayer patches with a free edge where lipid molecules arrange themselves into sharply curved and energetically unfavorable structures. ${ }^{11,47}$ The free energy per unit length from the edge grows as the vesicle ruptures and must be compensated by sufficient adhesion energy. As a consequence, large vesicles are more likely to rupture than smaller ones. This interplay between edge tension and adhesion energy agrees with previous studies ${ }^{10}$ and with our experimental observations that $100 \mathrm{~nm}$ vesicles rupture spontaneously (Figure 6).

This critical balance between edge tension and adhesion energy changes considerably as the vesicle size decreases. In the extreme case of isolated SUVs, spontaneous vesicle rupture is a rare event. Our observations suggest that the key to forming a bilayer from SUVs is the close proximity of several vesicles. Even though an isolated vesicle does not rupture, the presence of nearby vesicles causes the overall energetics to resemble those of one large vesicle and bilayer formation becomes favorable. Our experiments do not allow us to reveal the fundamental cause of vesicle rupture, but they demonstrate that bilayer edges that are inhibited vesicle rupture for isolated SUVs, now enhance the formation of the bilayer through the bilayer wave. In this scenario, vesicle rupture is highly cooperative and results in a wavelike bilayer propagation where a single rupture event initiates a chain reaction and leads to rapid SLB formation. The ratio of edge tension to total surface interaction energy improves as the patch grows, accelerating the formation of the bilayer (Figure 6E, Supporting Information, Movies S1-4). Eventually, the energetics become favorable enough that the bilayer edge not only catalyzes vesicle rupture, but also provides sufficient energy to 
physically push vesicles along the surface. The overall concept of cooperativity agrees with recent observations that a single vesicle rupture event can induce another one nearby for giant unilamellar vesicles. ${ }^{32}$

Throughout the acquisition of several tens of movies of bilayer formation on different substrates and with different vesicle sizes, we never observed clear signatures of vesicle fusion. The time traces shown in Figure 6C, D do not suggest that vesicle fusion plays a major role, because we predominantly observe positive and negative spikes of comparable magnitude and thus adsorbing and rupturing vesicles of similar size. If vesicle fusion was critical to bilayer formation, we would expect our time-traces to frequently exhibit larger desorption spikes as more material ruptures than initially adsorbed. Finally, wave formation often begins with the rupture of individual seed vesicles (Figure 5), but we did not observe any threshold in terms of a minimum vesicle size required to initiate wave-formation.

To conclude, we have demonstrated the unique ability of iSCAT to study the mechanism of SLB formation. Given the sensitivity of our technique, we believe that it will be an invaluable tool to eventually create a molecular-level understanding of the transition between nanoscopic vesicles and macroscopic lipid bilayers. From a practical perspective, iSCAT has the potential to become a new standard for evaluating the quality of artificial bilayers beyond fluidity alone from fluorescence recovery after photobleaching, by direct detection of trapped vesicles (Supporting Information, Movie S9). In addition to these implications for studies of and with SLBs, additional exciting prospects emerge from the presented work. The ability of iSCAT to detect and localize multiple particles down to $5 \mathrm{~nm}$ diameter ${ }^{35}$ within a diffraction limited spot without any degradation in image quality (Figure 5) makes the technique an excellent candidate for a super-resolution, non-fluorescence-based approach using the concept originally implemented by Hochstrasser and co-workers. ${ }^{48}$ Here, we studied a disassembly process on the nanoscale as vesicles transformed into a bilayer. The opposite is 
equally possible, where iSCAT can be used to study nanoparticle assembly with few-nm accuracy and simultaneous frame rates up to $100 \mathrm{kHz} .^{37}$

\section{MATERIALS AND METHODS}

\section{Glass cleaning and measurement chamber preparation}

Borosilicate glass cover glass (No. 1.5, 24 x $50 \mathrm{~mm}$, VWR) were cleaned by sequential sonication for 10 minutes each in water:isopropanol (50:50), Milli-Q water and $1 \mathrm{M}$ potassium hydroxide. They were then dried under a stream of dry nitrogen gas and exposed to UV/ozone for 8 minutes at $50 \mathrm{~W}$ power using a plasma cleaner (Diener Electronic, Plasma System Femto). All cover glass was used within one day of cleaning. To attach mica to the coverslip, index matched optical glue (Norland Optical Adhesive 61) was used. After the glue hardened (365 nm UV light for five minutes), mica layers were peeled off, leaving a thin layer of mica on the glass coverslip with its atomically flat side exposed. Generating a thin mica layer is important to minimize image deterioration caused by the birefringence of mica. CultureWell silicone gaskets (Grace Bio-Labs) were cut and placed onto the freshly cleaned coverslip or mica providing several independent 3-10 $\mu 1$ sample chambers on the same substrate.

\section{Sample preparation}

1,2-dioleoyl-sn-glycero-3-phosphatidylcholine (DOPC) in chloroform (Avanti Polar Lipids) was dried to a film, kept under vacuum for at least $1 \mathrm{~h}$ and brought to a concentration of 1 $\mathrm{mg} / \mathrm{mL}$ in bilayer buffer (10 mM Hepes, $\mathrm{pH}$ 6.8, $200 \mathrm{mM} \mathrm{NaCl}, 2 \mathrm{mM} \mathrm{CaCl} 2)$. Using a miniextruder (Avanti Polar Lipids), the suspension was then forced 21-times through a series of 
single membranes with decreasing pore sizes $-100 \mathrm{~nm} ; 100-50 \mathrm{~nm} ; 100-50-30 \mathrm{~nm}$, for 100 $\mathrm{nm}, 50 \mathrm{~nm}$ and $30 \mathrm{~nm}$ EUVs, respectively. Alternatively, $1 \mathrm{ml}$ of lipid solution was sonicated on ice for a total time of $60 \mathrm{~s}$ in $10 \mathrm{~s}$ intervals (amplitude $10 \mu \mathrm{m}$ ) using a MSE Soniprep 150 sonicator (Sanyo) and then centrifuged (10 minutes, $16000 \mathrm{~g}, 4^{\circ} \mathrm{C}$ ) to remove titanium particles. The vesicle size distribution was measured using a Viscotek 802 DLS and analyzed with OmniSIZE Software. SUV and EUV (100 nm extruded) hydrodynamic radii were found to be $10.5 \mathrm{~nm}+/-0.4 \mathrm{~nm}$ and $56.4 \mathrm{~nm}+/-6.2 \mathrm{~nm}$, respectively. SLBs were formed by the vesicle drop method ${ }^{49}$ by adding $4 \mu 1$ of buffer first to wet the substrate followed by $4 \mu 1$ of 1 $\mathrm{mg} / \mathrm{ml}$ DOPC solution.

\section{iSCAT}

The experimental setup and the technique have been described previously in detail. ${ }^{35,36}$ Briefly, we used a 60x 1.42 NA objective (Olympus) with a large back aperture and underfill the objective with the incoming beam to generate a relatively large focus of $1 \mu \mathrm{m}$. This has no effect on the observed iSCAT contrast, but leads to smoother illuminated areas $(30 \times 30$ $\mu \mathrm{m})$ when beam scanning. We found that active focus stabilization to within $20 \mathrm{~nm}$ using the reflection of an additional Helium-Neon laser improved the image quality. We also used a combination of polarizing beam splitter and quarter waveplate to extract the backscattered light from the inverted microcope more efficiently compared to the previous, less efficient approach of using a 50:50 beamsplitter. All movies were taken at $25 \mathrm{fps}$ with a camera exposure time of $30 \mathrm{~ms}$.

In contrast to many previous observations with iSCAT, here we often observed strong first interference in the point spread functions of adsorbed vesicles. We believe, that this is due to the combination of wide-field imaging and large particle contrast at an interface, a measurement that has not been reported previously. As a consequence of the coherent nature 
of the illumination source and the differences in the image formation process, iSCAT presents distinctive features that differentiate it from fluorescence. In fluorescence the image is formed with light from an incoherent source (fluorescence emitter), but in iSCAT the reflected and scattered light originates from a coherent source (laser). As a result, edge ringing is present in iSCAT both in the point spread function (PSF) of the optical system as well as in features with frequency components higher than the cut-off frequency imposed by the aperture of the objective. ${ }^{50}$

\section{AUTHOR INFORMATION}

\section{Corresponding Author}

*philipp.kukura@chem.ox.ac.uk

\section{Author Contributions}

The manuscript was written through contributions of all authors. All authors have given approval to the final version of the manuscript.

\section{Funding Sources}

This work was supported by the John Fell Fund and a career acceleration fellowship by the EPSRC EPSRC (EP/H003541). K.M.S. was supported by a fellowship from the National Institute of Biomedical Imaging and Bioengineering, National Institutes of Health (FEB013960).

\section{ACKNOWLEDGMENTS}

We thank H. Ewers and C. Eggeling for many helpful discussions. 


\section{SUPPORTING INFORMATION}

Movies of supported bilayer formation and individual file descriptions. This material is available free of charge via the internet at http://pubs.acs.org. 


\section{REFERENCES}

(1) Sackmann, E. Supported Membranes: Scientific and Practical Applications. Science 1996, 271, 43-48.

(2) Brian, A. A.; McConnell, H. M. Allogeneic Stimulation of Cytotoxic T Cells by Supported Planar Membranes. Proc. Natl. Acad. Sci. U. S. A. 1984, 81, 6159-6163.

(3) Hartman, N. C.; Nye, J. A.; Groves, J. T. Cluster Size Regulates Protein Sorting in the Immunological Synapse. Proc. Natl. Acad. Sci. U.S.A. 2009, 106, 12729-12734.

(4) Diao, J.; Ishitsuka, Y.; Lee, H.; Joo, C.; Su, Z.; Syed, S.; Shin, Y. K.; Yoon, T. Y.; Ha, T. A Single Vesicle-Vesicle Fusion Assay for in vitro Studies of SNAREs and Accessory Proteins. Nat. Protoc. 2012, 7, 921-934.

(5) Czolkos, I.; Jesorka, A.; Orwar, O. Molecular Phospholipid Films on Solid Supports. Soft Matter 2011, 7, 4562-4576.

(6) Nollert, P.; Kiefer, H.; Jahnig, F. Lipid Vesicle Adsorption versus Formation of Planar Bilayers on Solid-Surfaces. Biophys. J. 1995, 69, 1447-1455.

(7) Cremer, P. S.; Boxer, S. G. Formation and Spreading of Lipid Bilayers on Planar Glass Supports. J. Phys. Chem. B 1999, 103, 2554-2559.

(8) Richter, R. P.; Berat, R.; Brisson, A. R. Formation of Solid-Supported Lipid Bilayers: An Integrated View. Langmuir 2006, 22, 3497-3505.

(9) Keller, C. A.; Kasemo, B. Surface Specific Kinetics of Lipid Vesicle Adsorption Measured with a Quartz Crystal Microbalance. Biophys. J. 1998, 75, 1397-1402.

(10) Reviakine, I.; Brisson, A. Formation of Supported Phospholipid Bilayers from Unilamellar Vesicles Investigated by Atomic Force Microscopy. Langmuir 2000, 16, 1806-1815.

(11) Seifert, U.; Lipowsky, R. Adhesion of Vesicles. Phys. Rev. A 1990, 42, 4768-4771.

(12) Lipowsky, R.; Seifert, U. Adhesion of Vesicles and Membranes. Mol. Cryst. Liq. Cryst. $1991,202,17-25$.

(13) Seifert, U. Configurations of Fluid Membranes and Vesicles. Adv. Phys. 1997, 46, 13137.

(14) Keller, C. A.; Glasmästar, K.; Zhdanov, V. P.; Kasemo, B. Formation of Supported Membranes from Vesicles. Phys. Rev. Lett. 2000, 84, 5443-5446.

(15) Richter, R.; Mukhopadhyay, A.; Brisson, A. Pathways of Lipid Vesicle Deposition on Solid Surfaces: a Combined QCM-D and AFM Study. Biophys. J. 2003, 85, 3035-3047.

(16) Richter, R. P.; Brisson, A. R. Following the Formation of Supported Lipid Bilayers on Mica: a Study Combining AFM, QCM-D, and Ellipsometry. Biophys. J. 2005, 88, 3422-3433.

(17) Johnson, J. M.; Ha, T.; Chu, S.; Boxer, S. G. Early Steps of Supported Bilayer Formation Probed by Single Vesicle Fluorescence Assays. Biophys. J. 2002, 83, 3371-3379.

(18) Mashaghi, A.; Swann, M.; Popplewell, J.; Textor, M.; Reimhult, E. Optical Anisotropy of Supported Lipid Structures Probed by Waveguide Spectroscopy and Its Application to Study of Supported Lipid Bilayer Formation Kinetics. Anal. Chem. 2008, 80, 3666-3676.

(19) Edvardsson, M.; Svedhem, S.; Wang, G.; Richter, R.; Rodahl, M.; Kasemo, B. QCM-D and Reflectometry Instrument: Applications to Supported Lipid Structures and Their Biomolecular Interactions. Anal. Chem. 2009, 81, 349-361.

(20) Benes, M.; Billy, D.; Benda, A.; Speijer, H.; Hof, M.; Hermens, W. T. SurfaceDependent Transitions During Self-Assembly of Phospholipid Membranes on Mica, Silica, and Glass. Langmuir 2004, 20, 10129-10137.

(21) Reimhult, E.; Hook, F.; Kasemo, B. Intact Vesicle Adsorption and Supported Biomembrane Formation from Vesicles in Solution: Influence of Surface Chemistry, Vesicle Size, Temperature, and Osmotic Pressure. Langmuir 2003, 19, 1681-1691.

(22) Anderson, T. H.; Min, Y.; Weirich, K. L.; Zeng, H.; Fygenson, D.; Israelachvili, J. N. Formation of Supported Bilayers on Silica Substrates. Langmuir 2009, 25, 6997-7005. 
(23) Reimhult, E.; Hook, F.; Kasemo, B. Vesicle Adsorption on SiO2 and TiO2: Dependence on Vesicle Size. J. Chem. Phys. 2002, 117, 7401-7404.

(24) Reimhult, E.; Hook, F.; Kasemo, B. Temperature Dependence of Formation of a Supported Phospholipid Bilayer From Vesicles on SiO2. Phys Rev E 2002, 66, 051905.

(25) Cho, N. J.; Jackman, J. A.; Liu, M.; Frank, C. W. pH-Driven Assembly of Various Supported Lipid Platforms: A Comparative Study on Silicon Oxide and Titanium Oxide. Langmuir 2011, 27, 3739-3748.

(26) Hain, N.; Gallego, M.; Reviakine, I. Unraveling Supported Lipid Bilayer Formation Kinetics: Osmotic Effects. Langmuir 2013, 29, 2282-2288.

(27) Reimhult, E.; Larsson, C.; Kasemo, B.; Hook, F. Simultaneous Surface Plasmon Resonance and Quartz Crystal Microbalance with Dissipation Monitoring Measurements of Biomolecular Adsorption Events Involving Structural Transformations and Variations in Coupled Water. Anal. Chem. 2004, 76, 7211-7220.

(28) Weirich, K. L.; Israelachvili, J. N.; Fygenson, D. K. Bilayer Edges Catalyze Supported Lipid Bilayer Formation. Biophys. J. 2010, 98, 85-92.

(29) Reimhult, E.; Zach, M.; Hook, F.; Kasemo, B. A Multitechnique Study of Liposome Adsorption on Au and Lipid Bilayer Formation on SiO2. Langmuir 2006, 22, 3313-3319.

(30) Dimitrievski, K.; Zach, M.; Zhdanov, V. P.; Kasemo, B. Imaging and Manipulation of Adsorbed Lipid Vesicles by an AFM Tip: Experiment and Monte Carlo Simulations. Colloids Surf., $B$ 2006, 47, 115-125.

(31) Reviakine, I.; Rossetti, F. F.; Morozov, A. N.; Textor, M. Investigating the Properties of Supported Vesicular Layers on Titanium Dioxide by Quartz Crystal Microbalance with Dissipation Measurements. J. Chem. Phys. 2005, 122, 204711.

(32) Hamai, C.; Yang, T. L.; Kataoka, S.; Cremer, P. S.; Musser, S. M. Effect of Average Phospholipid Curvature on Supported Bilayer Formation on Glass by Vesicle Fusion. Biophys. J. 2006, 90, 1241-1248.

(33) Schonherr, H.; Johnson, J. M.; Lenz, P.; Frank, C. W.; Boxer, S. G. Vesicle Adsorption and Lipid Bilayer Formation on Glass Studied by Atomic Force Microscopy. Langmuir 2004, 20, 11600-11606.

(34) Lenz, P.; Johnson, J. M.; Chan, Y. H. M.; Boxer, S. G. Tension-Induced Pore Formation and Leakage in Adhering Vesicles. Europhys. Lett. 2006, 75, 659-665.

(35) Lindfors, K.; Kalkbrenner, T.; Stoller, P.; Sandoghdar, V. Detection and Spectroscopy of Gold Nanoparticles Using Supercontinuum White Light Confocal Microscopy. Phys. Rev. Lett. 2004, 93, 037401.

(36) Kukura, P.; Ewers, H.; Muller, C.; Renn, A.; Helenius, A.; Sandoghdar, V. High-Speed Nanoscopic Tracking of the Position and Orientation of a Single Virus. Nat. Methods 2009, 6, 923927.

(37) Ortega-Arroyo, J.; Kukura, P. Interferometric Scattering Microscopy (iSCAT): New Frontiers in Ultrafast and Ultrasensitive Optical Microscopy. Phys. Chem. Chem. Phys. 2012, 14, 15625-15636.

(38) Kukura, P.; Celebrano, M.; Renn, A.; Sandoghdar, V. Single-Molecule Sensitivity in Optical Absorption at Room Temperature. J. Phys. Chem. Lett. 2010, 1, 3323-3327.

(39) Ewers, H.; Jacobsen, V.; Klotzsch, E.; Smith, A. E.; Helenius, A.; Sandoghdar, V. LabelFree Optical Detection and Tracking of Single Virions Bound to Their Receptors in Supported Membrane Bilayers. Nano Lett. 2007, 7, 2263-2266.

(40) Mlodzianoski, M. J.; Juette, M. F.; Beane, G. L.; Bewersdorf, J. Experimental Characterisation of 3D Localization Techniques for Particle-Tracking and Super-Resolution Microscopy. Opt. Express 2009, 17, 8264-8277.

(41) Jacobsen, V.; Stoller, P.; Brunner, C.; Vogel, V.; Sandoghdar, V. Interferometric Optical Detection and Tracking of Very Small Gold Nanoparticles at a Water-Glass Interface. Opt. Express 2006, 14, 405-414. 
(42) Kukura, P.; Celebrano, M.; Renn, A.; Sandoghdar, V. Imaging a Single Quantum Dot When It Is Dark. Nano Lett. 2009, 9, 926-929.

(43) Krishnan, M.; Mojarad, N.; Kukura, P.; Sandoghdar, V. Geometry-Induced Electrostatic Trapping of Nanometric Objects in a Fluid. Nature 2010, 467, 692-695.

(44) Betzig, E.; Patterson, G. H.; Sougrat, R.; Lindwasser, O. W.; Olenych, S.; Bonifacino, J. S.; Davidson, M. W.; Lippincott-Schwartz, J.; Hess, H. F. Imaging Intracellular Fluorescent Proteins at Nanometer Resolution. Science 2006, 313, 1642-1645.

(45) Brokmann, X.; Coolen, L.; Hermier, J. P.; Dahan, M. Emission Properties of Single CdSe/ZnS Quantum Dots Close to a Dielectric Interface. Chem. Phys. 2005, 318, 91-98.

(46) Parker, J. L.; Cho, D. L.; Claesson, P. M. Plasma Modification of Mica - Forces between Fluorocarbon Surfaces in Water and a Nonpolar Liquid. J. Phys. Chem. 1989, 93, 6121-6125.

(47) Evans, E.; Rawicz, W. Entropy-Driven Tension and Bending Elasticity in CondensedFluid Membranes. Phys. Rev. Lett. 1990, 64, 2094-2097.

(48) Kuo, C. K.; Hochstrasser, R. M. Super-resolution Microscopy of Lipid Bilayer Phases. J. Am. Chem. Soc. 2011, 133, 4664-4667.

(49) Kalb, E.; Frey, S.; Tamm, L. K. Formation of Supported Planar Bilayers by Fusion of Vesicles to Supported Phospholipid Monolayers. Biochim. Biophys. Acta. 1992, 1103, 307-316.

(50) Considine, P. S. Effects of Coherence on Imaging Systems. J. Opt. Soc. Am. 1966, 56, 1001-1007. 